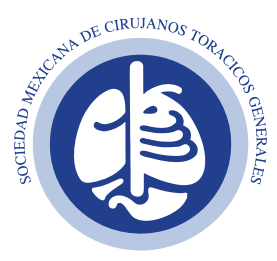

Vol. 1, Núm. 1

Enero-Abril 2020 pp 18-22

Palabras clave:

Metastasectomía, metástasis pulmonar, cuña, resección pulmonar, toracotomía abierta, cirugía torácica videoasistida.

Keywords: Metastasectomy, pulmonary metastasis, wedge, pulmonary resection, open thoracotomy, videoassisted thoracic surgery.

\footnotetext{
* Residente de 20 año de Cirugía General;

"Residente de 3er año de Cirugía General;

$\S$ Maestra adscrita de Servicio de Cirugía General;

II Maestro adscrito de Servicio de Cirugía Torácica y Cardiovascular;

** Jefe de Servicio de Cirugía General. Servicio de Cirugía General, Hospital Universitario José Eleuterio González.

₹ Presentado en el VII Congreso Internacional de la Sociedad Mexicana de Cirujanos Torácicos Generales en Monterrey Nuevo León.
}

Recibido: 05/02/2019

Aceptado: 18/04/2019

Correspondencia: Tania L Salazar-Islas E-mail: tania.salazarii@gmail.com
REVISTA

MEXICANA DE

CIRUGÍA

TORÁCICA

GENERAL

doi: $10.35366 / 94448$

https://dx.doi.org/10.35366/94448

\author{
Artículo original
}

\title{
Metastasectomía pulmonar: experiencia de cuatro años en el Hospital Universitario "Dr. José Eleuterio González"
}

\author{
Pulmonary metastasectomy: 4 years of experience at the \\ "Dr. José Eleuterio González" University Hospital
}

\author{
Tania Larissa Salazar-Islas, ${ }^{*}$ Héctor Hugo Romero-Garza, ${ }^{\natural}$ \\ Alethia Álvarez-Cano, \$ Manuel Wong-Jaén, || Gerardo Muñoz-Maldonado**
}

\section{RESUMEN}

Las metástasis pulmonares son un signo de enfermedad oncológica avanzada, y el pulmón es el segundo lugar más frecuente del organismo donde se presentan. La metastasectomía pulmonar es un procedimiento que cobra importancia para el control de la enfermedad oncológica al aumentar la sobrevida de los pacientes con cáncer. Los criterios actuales de selección para pacientes que son candidatos a una metastasectomía son: control del tumor primario o la posibilidad de resecar el tumor completo de forma simultánea con la metastasectomía, la capacidad de resección completa de las lesiones metastásicas, la capacidad del paciente de tolerar la resección pulmonar necesaria para remover por completo la tumoración, la ausencia de enfermedad extratorácica y la ausencia de tratamiento alternativo. La metastasectomía pulmonar se puede abordar mediante toracotomía abierta (TA) y cirugía videoasistida (VATS). Se presenta una serie de 13 casos que se analizaron de manera retrospectiva, en la que se evaluó el procedimiento llevado a cabo y el seguimiento posoperatorio, complicaciones y mortalidad, encontrando que en nuestra institución se reportó mayor número de días de estancia intrahospitalaria y complicaciones asociadas a un procedimiento abierto en comparación con la toracoscopia; sin embargo, la elección del tipo de procedimiento dependerá principalmente de la experiencia del cirujano con cada uno de dichos procedimientos. Además, una adecuada valoración preoperatoria optimizará la selección futura de candidatos a este procedimiento con el fin de lograr un impacto a favor de una mayor supervivencia.

\section{ABSTRACT}

Pulmonary metastases are a sign of advanced oncological disease and the lung is the second most frequent place in the organism where they occur. Pulmonary metastasectomy is a procedure that is important for the control of oncological disease by increasing the survival of patients with cancer. The current selection criteria for patients who are candidates for a metastasectomy are: control of the primary tumor or the possibility of resecting the complete tumor simultaneously with metastasectomy, the capacity for complete resection of metastatic lesions, the patient's ability to tolerate the pulmonary resection necessary to completely remove the tumor, the absence of extrathoracic disease and the absence of alternative treatment. Pulmonary metastasectomy can be approached through open thoracotomy (TA) and video assisted thoracic surgery (VATS). We present a series of 13 cases where the procedure carried out, the postoperative follow-up, complications and mortality were evaluated retrospectively. The results reported in our institution were: a greater number of days of in-hospital stay and complications associated with open thoracotomy compared to video assisted thoracoscopic surgery, however the choice of the type of procedure will depend mainly on the experience of the surgeon with each of these procedures. In addition, an adequate preoperative assessment will optimize the future selection of candidates for this procedure in order to achieve a greater impact in favor of survival.

Citar como: Salazar-Islas TL, Romero-Garza HH, Álvarez-Cano A, Wong-Jaén M, Muñoz-Maldonado G. Metastasectomía pulmonar: experiencia de cuatro años en el Hospital Universitario “Dr. José Eleuterio González". Rev Mex Cir Torac Gen. 2020; 1(1):18-22 


\section{INTRODUCCIÓN}

Las metástasis pulmonares son un signo de enfermedad oncológica avanzada, y el pulmón es el segundo lugar más frecuente del organismo donde se presentan. La metastasectomía pulmonar es un procedimiento que cobra importancia para el control de la enfermedad oncológica al aumentar la sobrevida de los pacientes con cáncer. ${ }^{1}$ Fue en 1938 que Barry y Churchill describieron por primera vez el caso de un paciente que sobrevivió 23 años después de una metastasectomía pulmonar por cáncer renal. Posteriormente, surgió una serie de reportes de casos que correlacionan la metastasectomía pulmonar con mayor sobrevida. ${ }^{2}$ Partiendo de ahí, se propusieron criterios básicos para la realización de las metastasectomías pulmonares; más tarde, estos criterios se han ido modificando hasta llegar a los que son actualmente aceptados.

La resección de lesiones metastásicas a pulmón se puede llevar a cabo por técnica abierta mediante toracotomía o a través de cirugía videoasistida. La elección del tipo de procedimiento dependerá principalmente del número y la localización de las lesiones reportadas, la función ventilatoria del paciente y la experiencia del cirujano con cada uno de dichos procedimientos.

El objetivo principal de este trabajo es analizar los casos de manera retrospectiva en quienes se llevó a cabo una metastasectomía pulmonar y evaluar la evolución posoperatoria de dichos pacientes de acuerdo con el procedimiento realizado, ya sea toracoscopia videoasistida (VATS) o una toracotomía, tomando en cuenta los días de estancia intrahospitalaria posteriores a la cirugía, las complicaciones posquirúrgicas y la mortalidad asociada al procedimiento; además de la identificación de factores pronósticos para optimizar la selección futura de candidatos a este tipo de procedimiento, así como el seguimiento realizado para valorar la recurrencia de la enfermedad y el impacto de la cirugía.

\section{MATERIAL Y MÉTODOS}

Se utilizó una base de datos retrospectiva a cuatro años, en la cual se incluyeron todos los pacientes que fueron sometidos a metastasectomías pulmonares, sin distinción de género o edad. Los datos fueron procesados y analizados por medio del paquete IBM SPSS Statistics 23. Se utilizaron pruebas de normalidad para caracterizar la distribución de los datos: los datos paramétricos serán reportados en términos de media (desviación estándar) y los no paramétricos como mediana (rango intercuartil). Se utilizaron pruebas de $\chi^{2}$ y test exacto de Fisher, así como de Wilcoxon para la comparación de grupos.

Se recopiló un total de 13 expedientes que cumplían con la información necesaria para la base de datos a realizar. Se tomó en cuenta información acerca del paciente como edad, género, el diagnóstico oncológico, el procedimiento aplicado, su evolución intrahospitalaria y el alta. Se valoró la histología de la tumoración primaria, la etapa clínica y la presencia de metástasis pulmonares al momento del diagnóstico de la enfermedad, así como la presencia de nuevas lesiones identificadas durante el seguimiento y la necesidad de quimioterapia neoadyuvante. En lo referente al procedimiento, se discrimina entre VATS o cirugía abierta, y su evolución intrahospitalaria, días de estancia posterior a cirugía, complicaciones perioperatorias y la mortalidad asociada al procedimiento quirúrgico. Además, se valoró la mortalidad global, incluyendo la duración de su seguimiento posterior al egreso.

\section{RESULTADOS}

En retrospectiva, se analizaron los casos de los expedientes mencionados, siendo siete pacientes mujeres, con un rango de edad para la serie de entre 12-71 años, y una mediana de 59 años (Tabla 1). En cuanto el diagnóstico histológico se detectó cáncer de colon (2), sarcoma de tejidos blandos (2), osteosarcoma (2), cáncer de endometrio (2), cáncer de mama (2), melanoma (2) y cáncer renal (1). Se observó que el $23.1 \%$ (3) de los pacientes ya presentaban metástasis pulmonares al momento del diagnóstico, y el resto desarrolló lesiones metastásicas a pulmón durante su seguimiento en un plazo promedio de 12 meses, observando un rango desde uno hasta 60 meses libres de enfermedad metastásica. Se prescribió quimioterapia neoadyuvante en el $76.9 \%$ de los casos como parte del protocolo realizado por el Servicio de Oncología médica a pacientes con metástasis candidatos a cumplir con los criterios de resección de lesiones metastásicas.

Del total de los 13 procedimientos quirúrgicos se efectuaron nueve metastasectomías por VATS y cuatro por toracotomía abierta. Dentro del análisis de la información se obtuvo una tendencia a estancia intrahospi- 
Tabla 1: Tabla de resultados.

\section{Variables}

Sexo, $n(\%)$

Edad, media, mediana

Tumor primario

Período libre de enfermedad, media

Metástasis iniciales (M1), $n$ (\%)

QT neoadyuvante, $n(\%)$

Toracotomías, $n$ (\%)

VATS, $n(\%)$

Días de estancia intrahospitalaria, media, mediana

Complicaciones, $\mathrm{n}(\%)$

Mortalidad global, $n(\%)$

Perioperatoria, $n(\%)$

Durante seguimiento, $\mathrm{n}(\%)$
Resultados

Fem 7 (53.8) Masc 6 (46.2)

50 años; 59 años (12-71 años)

9 estirpes histológicas

12 meses (0-60 meses)

$3(23.1)$

$10(76.9)$

$4(30.8)$

$9(69.2)$

9 días; 5 días (3-30 días)

$4(30.8)$

$4(30.8)$

$3(23.1)$

$1(7.6)$ talaria prolongada en pacientes en quienes se realizó una toracotomía en comparación con VATS, con una diferencia estadística significativa ( $p=0.041)$, con una media de estancia intrahospitalaria posoperatoria de 4 $( \pm 1)$ días por VATS y 20 días ( \pm 10 ) por cirugía abierta; además de corresponder a una mayor tasa de complicaciones asociadas que resultó en la prolongación de su internamiento. En cuanto a las complicaciones hubo cuatro casos, tres de ellas en pacientes posoperados de cirugía abierta y un caso en quien se realizó toracoscopia con una clara tendencia a la asociación con la cirugía abierta en comparación con la cirugía videoasistida ( $p=0.085$ ). Entre las complicaciones posoperatorias que se presentaron posteriores a una cirugía abierta están: infección de herida quirúrgica, tromboembolismo pulmonar y empiema. Sin embargo, la única complicación por VATS que se reportó fue una hemorragia transoperatoria que evolucionó a choque hipovolémico y defunción (Tabla 2). En general, se observó una mortalidad perioperatoria del $23.1 \%{ }^{3}$ No se encontró asociación estadística entre el procedimiento realizado y la mortalidad ( $p=0.462)$.

Hubo recurrencia de la enfermedad en el $30.8 \%$ de los casos durante el seguimiento de los pacientes. El tiempo de seguimiento de los pacientes quienes se sometieron a la resección de las metástasis varía, debido a que se trata de un estudio retrospectivo desde seis meses, que fue el último caso que se llevó a cabo, hasta 48 meses, que fue el primer caso de esta serie. Se reportó un $7.6 \%{ }^{1}$ de mortalidad durante el
Tabla 2: Complicaciones y resultados.

\begin{tabular}{cl} 
& \multicolumn{1}{c}{ Complicaciones } \\
\hline \multicolumn{1}{c|}{ VATS } & \multicolumn{1}{c}{ Toracotomía } \\
\hline $\begin{array}{l}\text { Hemorragia } \\
\text { transoperatoria (1) }\end{array}$ & $\begin{array}{l}\text { Infección de la herida quirúrgica (1) } \\
\text { Empiema (1) } \\
\text { Tromboembolismo pulmonar (1) }\end{array}$ \\
\hline & Mortalidad \\
\hline 1 & 2 \\
\hline
\end{tabular}

seguimiento de los pacientes, que en conjunto con la mortalidad perioperatoria nos llevó a una mortalidad global del $30.8 \%{ }^{4}$

\section{DISCUSIÓN}

Las metástasis pulmonares de una lesión maligna extrapulmonar son una manifestación de enfermedad oncológica avanzada. La metastasectomía pulmonar es una opción para su control. Resecar las metástasis permite prolongar la sobrevida, inclusive dejar libre de enfermedad oncológica al paciente. ${ }^{1}$ La resección agresiva de las metástasis se ha ido convirtiendo en una práctica cada vez más aceptada. Las metástasis pulmonares raramente tienen manifestaciones sintomáticas, es frecuente que sean diagnosticadas en los estudios de extensión de otra enfermedad oncológica primaria. En los pacientes con 
síntomas como tos, dolor y hemoptisis, se presentan en las lesiones hiliares, mientras que las lesiones periféricas inician con neumotórax espontáneo. ${ }^{3}$

Para llevar a cabo este tipo de procedimientos, se requiere de un equipo interdisciplinario, que incluya al médico oncólogo y al cirujano torácico, que aborde al paciente desde diferentes perspectivas y así obtener la mejor opción de tratamiento para lograr beneficiar al paciente. Esto con el objetivo de obtener resultados que sustenten la aplicación metódica de nuevas líneas de manejo para estadíos de enfermedad avanzada, lo que nos orientará a establecer los criterios necesarios para llevar a cabo este procedimiento con intención curativa.

Los criterios actuales de selección para pacientes que son candidatos a una metastasectomía son: control del tumor primario o la posibilidad de resecar el tumor completo de forma simultánea con la metastasectomía, la capacidad de resección completa de las lesiones metastásicas, la capacidad del paciente de tolerar la resección pulmonar necesaria para remover por completo la tumoración, la ausencia de enfermedad extratorácica y la ausencia de tratamiento alternativo. ${ }^{2}$ Algunos factores pronósticos desfavorables para la metastasectomía serían que no se tuviera la capacidad de resecar por completo las lesiones, breve período libre de enfermedad después del manejo del tumor primario, múltiples metástasis pulmonares, involucro de ganglios linfáticos, así como la histología del tumor. ${ }^{1}$ En uno de los casos se realizó una metastasectomía por cirugía abierta a un paciente con un período menor de seis meses libre de enfermedad posterior a una amputación transhumeral por un diagnóstico de mixofibrosarcoma; sin embargo, un mes después inició con manifestaciones neurológicas y se evidenciaron lesiones metastásicas a sistema nervioso central. El paciente recibió quimioterapia paliativa y falleció dos meses después. He aquí la importancia de la estirpe histológica del tumor, saber que el sarcoma pleomórfico tiene un potencial metastásico elevado, así como un breve período libre de enfermedad como factor pronóstico de recidiva nos ayudarían a una mejor selección de pacientes.

Aún se continúa con la búsqueda de otros factores pronósticos que mejoren la selección de pacientes, de tal manera que nos conduzca a una cirugía más segura y efectiva. Algunos de los factores que actualmente se continúan estudiando son: estirpe histológica, período libre de enfermedad, presencia de metástasis al momento del diagnóstico y el número de nódulos pulmonares.
La metastasectomía pulmonar se puede abordar mediante toracotomía abierta (TA) y cirugía videoasistida (VATS). La toracotomía se consideraba el estándar de manejo en los pacientes con metástasis pulmonares. $^{2}$ Con la VATS se ha observado una mejoría en el dolor posquirúrgico y mejor preservación de la función pulmonar. ${ }^{2}$ McCormack et al. realizaron un estudio prospectivo evaluando el rol de la VATS para tratar las metástasis pulmonares, 18 pacientes tenían un TAC previo al procedimiento quirúrgico, en el cual se efectuó la resección de todas las lesiones visibles y detectadas por TAC. Todos los pacientes fueron sometidos a toracotomía, por medio de la cual se hizo la resección de lesiones agregadas. Se encontraron lesiones agregadas en el $56 \%$ de los pacientes. ${ }^{4}$ En la revisión de Internullo $\mathrm{E}$, et al. se identifica que el $67 \%$ de los cirujanos consideran necesaria la palpación para la identificación de lesiones metastásicas, por lo que en ese estudio se concluye que la VATS está indicada en pacientes con lesiones bien definidas por estudios de imagen o en pacientes con comorbilidades asociadas, en quienes se considere la toracotomía como un procedimiento de alto riesgo. ${ }^{5}$ Sin embargo, con el surgimiento de estudios de imagen cada vez con mayor resolución se ha identificado mayor número de lesiones en etapas más tempranas, haciendo de la VATS el procedimiento de elección en centros de alto volumen.

\section{CONCLUSIONES}

La metastasectomía pulmonar se ha realizado con intención curativa con resultados asociados a un período mayor de supervivencia, con recurrencia de la enfermedad en menos del $30 \%$ de los casos presentados, así como una evolución posoperatoria con menos complicaciones y estancia intrahospitalaria más corta por VATS en comparación con la técnica abierta. Se observó una relación significativa entre el procedimiento aplicado y el tiempo de estancia hospitalaria, además de una tendencia a mayor número de complicaciones y mortalidad asociadas con un procedimiento abierto. No se encontró asociación entre el período libre de enfermedad, metástasis iniciales y el uso de quimioterapia neoadyuvante con la mortalidad o recurrencia de la enfermedad. Por otra parte, una adecuada valoración preoperatoria optimizará la selección futura de candidatos a este procedimiento con el fin aumentar la supervivencia. 


\section{REFERENCIAS}

1. Pastorino U. Long-term results of lung metastasectomy: prognostic analyses based on 5206 cases. J Thorac Cardiovasc Surg. 1997; 113(1): 37-49. doi: 10.1016/S0022-5223(97)70397-0.

2. Ripley RT, Rush VW. Lung metastases. In: Abeloff's clinical oncology. 5th ed. Philadelphia, PA: Churchill Livingstone Elsevier; 2014. pp. 764-777.

3. Srinivas S, Varadhachary G. Spontaneous pneumothorax in malignancy: a case report and review of the literature. An Oncology. 2000; 11(7): 887-889. doi: 10.1023/a:1008323632078.
4. Mccormack PM, Ginsberg KB, Bains MS, Burt ME, Martini N, Rusch VW et al. Accuracy of lung imaging in metastases with implications for the role of thoracoscopy. An Thorac Surg. 1993; 56(4): 863-866. doi: 10.1016/0003-4975(93)90344-h.

5. Internullo E, Cassivi SD, Raemdonck DV, Friedel G, Treasure T. Pulmonary metastasectomy: a survey of current practice amongst members of the european society of thoracic surgeons. J Thorac Oncol. 2008; 3(11): 1257-1266. doi: 10.1097/jto.0b013e31818bd9da.

Conflicto de intereses: Ninguno. 